\section{Candidate drug for ALS}

There is currently only one approved treatment for amyotrophic lateral sclerosis (ALS), and phase III trials for two new compounds recently failed. Thus, there is a substantial need to test potential new drugs with rigorous in vitro models before testing in the clinic. Now, Lee Rubin and colleagues report a small molecule screen in stem cell-derived motor neurons in which they identified the GSK-3 inhibitor kenpaullone as a candidate therapeutic for ALS (Cell Stem Cell 12, 713-726, 2013). The authors differentiated motor neurons from wild-type mouse embryonic stem cells (ESCs) and ESCs from mice carrying the SOD1G93A human transgene. They screened a library of 5,000 small molecules and identified a number of compounds that improved survival of one or both types of motor neurons, including several kinase inhibitors. The authors further investigated kenpaullone, which reproducibly increased the survival of both wild-type and SOD1 $1^{\mathrm{G} 93 \mathrm{~A}}$ motor neurons. Other known GSK-3 inhibitors had weaker or no effect, suggesting that the effect of kenpaullone might be due to effects besides GSK-3 inhibition. Using an antibody array for phosphorylated kinases, the authors discovered that kenpaullone reduces the phosphorylation and activation of the JNK-c-Jun cell death pathway. Kenpaullone also promoted the survival of motor neurons differentiated from human ALS induced pluripotent stem cells.

\section{MKRN3 and pubertal timing}

Onset of puberty is marked by pulsatile release of gonadotropinreleasing hormone $(\mathrm{GnRH})$ and is thought to be influenced by genetic factors. Now, Ana Claudia Latronico and colleagues report the identification of genetic mutations that cause familial precocious puberty $(N$. Engl. J. Med. doi:10.1056/NEJMoa1302160, 5 June 2013). The authors performed exome sequence analysis of individuals from 15 families with central precocious puberty and identified heterozygous truncating and missense mutations in MKRN3 segregating in 5 families. MKRN3 is a paternally expressed imprinted gene located on chromosome 15 in the Prader-Willi syndrome locus, and all affected individuals in these families inherited the causative mutation from their fathers. MKRN3 encodes makorin RING-finger protein 3, but the function of this protein is not known. The authors also examined mouse Mkrn3 expression in the arcuate nucleus of the hypothalamus, where other genes with functions in pubertal timing are expressed. They showed that expression of Mkrn3 is high during development and decreases immediately before puberty. This work provides new insight into pathways involved in pubertal timing.

\section{Heritability estimates}

Alkes Price and colleagues report estimates of the narrow-sense heritability $\left(h^{2}\right)$ explained for 23 complex phenotypes, including 11 quantitative and 12 dichotomous traits (PLoS Genet. 9, e1003520, 2013). They analyzed 300,000 SNPs genotyped in 38,167 individuals from Iceland, including long-range phasing, combined with an extensive genealogical database. Their heritability estimates were smaller than those previously

Written by Pamela Feliciano, Emily Niemitz, Orli Bahcall \& Kyle Vogan reported, which have often been based on comparisons between closely related individuals. In contrast, the authors developed a new identity by descent (IBD)-based approach that allows for heritability estimation using data from both closely and distantly related pairs of individuals. Their linear mixed-model approach allows for joint estimation of $h^{2}$ and the fraction of heritability explained by SNPs genotyped in genomewide association studies (GWAS), facilitating a direct comparison of these estimates using the same data sets. The authors also examined the effects of shared environment, dominance and epistasis and found that shared environment was the major contributor to the inflation of $h^{2}$ estimates observed in data sets including closely related individuals. The authors are also more optimistic than those of previous reports in terms of expectations for the fraction of heritability explained by common variation, noting that over half of $h^{2}$ may be explained by SNPs on genotyping platforms used in GWAS.

$O B$

\section{Congenital neutropenia and VPS45}

Two independent studies have identified recessive mutations in the endosomal trafficking gene VPS45 as the cause of a rare congenital syndrome characterized by severe neutropenia, bone marrow fibrosis, nephromegaly and susceptibility to life-threatening bacterial infections. Dror Mevorach and colleagues (Blood doi:10.1182/blood-201212-475566, 22 April 2013) performed homozygosity mapping and exome sequencing on two Palestinian families with this syndrome and identified a homozygous missense mutation in VPS45 present in both families. Raz Somech and colleagues (N. Engl. J. Med. doi:10.1056/ NEJMoa1301296, 5 June 2013) performed similar analyses on four Palestinian families and identified the same homozygous missense mutation in VPS45 in all four families. They also analyzed a fifth affected family from Morocco and identified a different homozygous missense mutation in VPS45. Both groups also analyzed cells from affected individuals and observed defects in intracellular trafficking and increased rates of apoptosis. VPS45 encodes a protein that interacts with multiple components of the endocytic and secretory pathways. The findings underscore the sensitivity of neutrophils and other hematopoietic lineages to perturbations in VPS45-mediated intracellular trafficking.

KV

\section{A secreted PTEN}

PTEN is a known tumor suppressor in several types of cancer and also antagonizes the phosphoinositide 3-kinase (PI3K) pathway. By analysis of the PTEN mRNA transcript, Ramon Parsons and colleagues found an alternative initiation codon that was $\sim 500$ bp upstream of and in frame with the typical initiation codon (Science doi:10.1126/science.1234907, 6 June 2013). They named the encoded variant PTEN-Long, which contains an additional 173 amino acids at its $\mathrm{N}$ terminus and also contains a secretion signal sequence at amino acid 22 . They found that PTEN-Long is secreted from cells and can decrease PI3K signaling exogenously. They then tested whether PTEN-Long could reduce tumor burden in mice and found that intraperitoneal injection of PTEN-Long into mice carrying xenograft tumors from several human cancer cell lines led to tumor regression. The authors suggest that recombinant PTEN-Long may be useful for antagonizing the PI3K pathway in the clinic. 